
allemande

45-2 | 2013

Images et discours de la nation

\title{
Richard FABER, Uwe PUSCHNER (dir.), Intellektuelle und Antiintellektuelle im 20. Jahrhundert
}

Berne, Peter Lang, 2013

Anne-Laure Briatte-Peters

\section{CpenEdition}

Journals

Édition électronique

URL : https://journals.openedition.org/allemagne/1801

DOI : 10.4000/allemagne.1801

ISSN : 2605-7913

Éditeur

Société d'études allemandes

Édition imprimée

Date de publication : 30 décembre 2013

Pagination : 492-495

ISSN : 0035-0974

Référence électronique

Anne-Laure Briatte-Peters, «Richard FABER, Uwe puschner (dir.), Intellektuelle und Antiintellektuelle im 20. Jahrhundert », Revue d'Allemagne et des pays de langue allemande [En ligne], 45-2 | 2013, mis en ligne le 29 juillet 2019, consulté le 23 mai 2021. URL : http://journals.openedition.org/allemagne/1801 ; DOI : https://doi.org/10.4000/allemagne.1801 
1796 en France dans les bureaux de bienfaisance: à Strasbourg ou Nancy, les trois cultes reconnus étaient représentés dans leurs commissions administratives. Pourtant, le dernier tiers du siècle vit se multiplier les conflits entre assistance municipale et bienfaisance catholique: en Prusse, les autorités accordaient leur préférence aux initiatives protestantes; à Strasbourg, les fonctionnaires du Reichsland se méfiaient de la Société de Saint-Vincent-de-Paul dont le siège était à Paris. En France, l'offensive laïque contre l'influence cléricale dans l'action sanitaire et sociale fut violente à partir de 1880. Le remplacement des salles d'asile par les écoles maternelles permit d'éliminer le personnel congréganiste. Cette tension ne fut quand même pas constante: les deux partenaires avaient trop souvent besoin l'un de l'autre pour se séparer complètement. Fait significatif, à Strasbourg, en 1906, Weydmann, jusqu'alors secrétaire de la Fédération de Charité catholique, fut appelé par le maire libéral Schwander à diriger le service d'assistance publique de la ville.

En terminant, Catherine Maurer constate que la comparaison entre les deux pays lui a permis de mettre en évidence les limites du modèle national de société: le caractère international du catholicisme a produit des pratiques convergentes. De part et d'autre, les créateurs d'ceuvres reprirent l'image pessimiste de la société moderne "comme un corps malade", ce qui ne les empêcha pas de tenter de trouver des réponses à plusieurs aspects de la modernité sociale, ne serait-ce que pour réagir aux défis qui menaçaient la survie de la religion.

On a scrupule à formuler des critiques, mineures il est vrai, devant l'ampleur et la solidité de ce travail. II me semble qu'il vaut mieux éviter l'usage de sigles et acronymes pour désigner des institutions du xixe siècle, époque où leur emploi était bien plus rare que de notre temps. On peut aussi s'étonner de voir l'adjectif «inquisitorial » désigner ce qui relève de l'enquête socio-historique: il évoque pour le lecteur français des "enquêtes » plus brutales. De façon plus globale, le lecteur garde le regret de ne pas en apprendre plus sur la sociologie et la psychologie des bénéficiaires de l'assistance confessionnelle et aussi de ceux qui en étaient exclus. Dans une recherche centrée sur les villes, on souhaite aussi connaître davantage sur la place de ces déshérités dans l'univers urbain, leur visibilité et évidemment leur pratique ou non de la religion. Mais il s'agit là d'un autre travail qui relève, lui du domaine de l'histoire sociale de la pauvreté et des détresses, tel qu'il a été entamé pour la France par André Gueslin.

Léon STRAUSS

Richard FABER, Uwe PUSCHNER (dir.), Intellektuelle und Antiintellektuelle im 20. Jahrhundert, Berne, Peter Lang (Zivilisationen \& Geschichte; 20), 2013, 239 p., 46,95€.

Jean-Paul Sartre, peut-être la dernière incarnation du modèle dominant de l'intellectuel depuis l'affaire Dreyfus, nous a quittés en 1980; tout se passe, depuis, comme si les chercheurs de plusieurs disciplines des sciences humaines et sociales avaient repris le flambeau pour œuvrer à la définition de ce que doit être ou de ce qu'est un intellectuel. Alors que plus d'un avait constaté son silence, voire annoncé sa mort, cette figure sociale est l'objet de discussions des plus vivantes. De fait, en Allemagne, en France et jusqu'aux États-Unis, la recherche sur les intellectuels connaît un fort regain d'intérêt depuis trois décennies; en témoignent en Allemagne de nombreuses publications récentes. Citons par exemple la monographie de Dietz Bering, Die Epoche der Intellektuellen 1889-2001. Geburt, Begriff, Grabmal, 2010 ( $2^{e}$ éd. 2011), deux ouvrages collectifs: Jutta Schlich (dir.), Intellektuelle im 20. Jahrhundert in Deutschland. Ein Forschungsreferat (2000) et Thomas Kroll, Tilman Reitz (dir.), Intellektuelle in der Bundesrepublik Deutschland. 
Verschiebungen im politischen Feld der 1960er und 1970er Jahre (2013), ainsi qu'un article publié en édition électronique de Daniel Morat: "Intellektuelle und Intellektuellengeschichte », in: Docupedia-Zeitgeschichte, 20.11.2011.

Le présent ouvrage collectif, qui s'inscrit dans cette discussion scientifique internationale et transdisciplinaire, émane d'une série de conférences organisées en 2010 par Richard Faber, professeur en sociologie de la culture, à l'Université libre de Berlin sur le thème "Qu'est-ce qu'un intellectuel? 》Ce recueil d'articles se présente comme le fruit de réflexions sur la transformation de la figure sociale de l'intellectuel; ce faisant, il met en évidence la fluidité des frontières entre intellectuels et anti-intellectuels. Organisé de façon symétrique, l'ouvrage commence par une contribution qui replace la naissance de l'intellectuel, avec l'affaire Dreyfus, dans le contexte de l'histoire des intellectuels. Deux parties se font vis-à-vis: l'une, simplement intitulée "intellectuels ", regroupe quatre contributions sur des intellectuels allemands et étrangers, l'autre, intitulée "anti-intellectuels", présente quatre exemples d'anti-intellectualisme. Revenant sur le questionnement initial, l'ouvrage se clôt sur un article qui analyse les tentatives actuelles de redéfinir la fonction de l'intellectuel.

Placé en exergue de l'ouvrage, l'article de l'historienne Anne Kwaschik met en perspective le modèle de l'intellectuel qui a dominé tout le xxe siècle en revenant sur sa naissance à l'occasion de l'affaire Dreyfus. S'appuyant sur la tradition française de l'histoire des intellectuels, qui définit le cadre de l'intervention intellectuelle au croisement entre milieu, lieu et réseau, elle revient sur les mobilisations en amont de ce qui allait devenir l'" affaire » ainsi que sur les conditions d'intervention d'Émile Zola. Il apparaît que l'intellectuel s'appuie sur des réseaux de personnes et de communications - les "sociabilités intellectuelles 》 (Racine et Trébitsch, 1992) - dans lesquels il endosse le rôle de figure de proue. Une analyse de discours incluant des éléments de dramaturgie révèle la dimension performative de l'intervention intellectuelle, lorsque, fort de sa notoriété publique, l'intellectuel se pose en autorité morale indépendante, en défenseur de la vérité et de valeurs universelles. Telles seraient les origines de la définition caractéristique de l'historiographie française des intellectuels, articulant la dimension individuelle et collective, ainsi que la portée universelle de l'intervention intellectuelle.

Dans la partie dédiée aux «intellectuels », la contribution d'Helen Theil sur la conception de l'intellectuel selon Simone Weil fournit un exemple d'une certaine forme d'antiintellectualisme chez "l'intellectuelle Simone Weil » (p. 47). Rejetant la fonction sociale exclusive de l'intellectuel parce que non démocratique et élitiste, Simone Weil appelait de ses voux une intellectualisation de la société, de chaque individu en elle. Dans cette société idéale, où chaque individu est doué et capable de raison, chacun est responsable de ses actes, au travail comme en politique, et peut ainsi se passer des intermédiaires qui l'empêchent de faire un usage propre de sa raison: l'expert, l'homme politique, le parti. Simone Weil attend des intellectuels une indépendance en toutes circonstances. Or, du moment qu'ils interviennent en tant que tels, ils sont, selon elle, déjà gagnés à une cause ou à un parti, perdant ainsi leur autonomie et leur attachement aux valeurs universelles. Le portrait du médecin, auteur et communiste militant Friedrich Wolf que propose I'historien Jens Flemming illustre ce phénomène. Friedrich Wolf est un auteur prolifique, qui produit des pièces et romans à thèse, un auteur engagé s'il en est. Aux antipodes de l'intellectuel retiré du monde, il fait la chronique des conflits sociaux et bouleversements révolutionnaires de son siècle, porté par l'idéologie communiste à laquelle il n'a jamais cessé d'adhérer. Jens Flemming voit en lui un infatigable auteur engagé, un "activiste littéraire » ( $p .77)$, qui intervenait au nom d'une autorité intellectuelle qu'il s'attribuait, mais non un intellectuel au sens du détenteur d'une autorité morale indépendante, sans attache partisane. Rüdiger Hentschel dresse un portrait de l' intellectuel Rudi Dutschke», en reconstruisant son parcours intellectuel à partir de ses lectures et interprétations des textes de Lukas, mais aussi d'analyses de Paul Sering sur le capitalisme moderne et de Sawinkow sur l'organisation du combat révolutionnaire. Apparaît ainsi une évolution: 
d'abord membre charismatique de l'" Action subversive » et du SDS (I'Union allemande des étudiants socialistes) porté par un marxisme inébranlable, Rudi Dutschke a plus tard formulé son rejet du terrorisme d'extrême gauche de la RAF (Fraction Armée Rouge), pour finalement se faire le défenseur d'une lutte des classes émancipatrice, dans laquelle les intellectuels joueraient un rôle indispensable. Originaire de la RDA, qu'il avait quittée avant la construction du Mur, Dutschke serait resté en RFA un intellectuel inclassable parmi les intellectuels ouest-allemands. Ehrhard Stölting retrace quant à lui l'histoire des intellectuels en Russie, de la constitution progressive de ce groupe social à partir du XVIII siècle jusqu'à nos jours. Il le fait dans un mouvement de va-et-vient entre les intellectuels occidentaux et l'intelligenzija russe, et constate ainsi l'existence d'analogies structurelles entre ces deux groupes. Examinant la validité des topoi relatifs aux intellectuels occidentaux pour le cas de la Russie, Ehrhard Stölting relève plusieurs caractéristiques de l'intelligenzija russe, en particulier sa rigueur morale, d'où se dégagent en creux des caractéristiques des intellectuels occidentaux.

La galerie de portraits rassemblés sous le titre "anti-intellectuels", dans la deuxième partie de l'ouvrage, ne compte que des personnalités allemandes. Richard Faber illustre les liens étroits qui peuvent exister entre modernisme et anti-modernisme, intellectualisme et anti-intellectualisme à l'exemple du germaniste et écrivain Karl Wolfskehl, disciple de Stefan George et pilier de la Bohême munichoise. Dans un portrait haut en couleurs, il met en évidence les paradoxes du personnage - par exemple entre le culte de la germanité et l'affirmation de son judaïsme dans un entourage de plus en plus ouvertement antisémite - et son syncrétisme apparemment sans limites. Germaniste chevronné et esprit quasi universel, aussi bien que sombre ésotériste, Karl Wolfskehl est, selon Richard Faber, intellectuel et anti-intellectuel dans une égale mesure. Partant de l'aversion des artisans de l'idéologie völkisch pour les intellectuels autant que pour la civilisation occidentale, la culture des grandes villes et les transformations de la société industrielle, Uwe Puschner s'interroge sur l'existence d'un type d'intellectuels völkisch, là où la conception classique de l'intellectuel place celui-ci résolument à gauche. II présente pour cela le biologiste et théoricien völkisch Willibald Hentschel et son audacieux "plan Mittgart » (Mittgart ou Midgard, litt. l'enceinte du milieu; ce terme est emprunté à la mythologie nordique), projet de colonie d'hommes et de femmes sélectionnés selon des critères raciaux à des fins de préservation et de culture de la race germanique. En dépit des capacités intellectuelles de Hentschel, de l'usage qu'il fait du verbe au service des idées et valeurs qu'il défend, de l'engagement continu de sa personnalité pour cette cause à ses yeux supérieure, Uwe Puschner lui refuse le statut d'intellectuel au sens propre en raison du caractère figé de sa pensée, de son incapacité à l'auto-critique et du peu d'influence qu'il soit jamais parvenu à exercer sur ses contemporains. Relevant du type du démagogue, du fanatique et du prophète séculaire, Hentschel renverrait plutôt l'image d'une déformation de l'intellectuel. Le germaniste Ralf Georg Czapla analyse les manifestations de l'anti-intellectualisme chez Joseph Goebbels, qui, nommé en 1933 ministre du Reich à l'Éducation du peuple et à la Propagande, contrôlait pourtant l'ensemble du secteur culturel et artistique. Cet anti-intellectualisme était construit sur un antagonisme entre l'intellect et les sentiments, caractéristique de l'idéologie völkisch, combiné à une forte valorisation de l'irrationnel. Partant de la biographie, du parcours universitaire et des échecs professionnels de Goebbels dans l'écriture et l'analyse littéraire, Czapla s'appuie sur la psychologie individuelle pour formuler son interprétation des origines de l'anti-intellectualisme virulent de Goebbels. Le politologue Michael Kohlstruck présente, en la personne du journaliste, universitaire et auteur Klaus Mehnert, un "intellectuel pour non intellectuels». Mehnert n'est pas un anti-intellectuel dans le même sens que les idéologues völkisch ci-dessus; sa seule profession de journaliste, puis de professeur d'université, fait de lui un intellectuel. Mais c'est plutôt par l'autorité qu'il a acquise au fil de ses publications, qui s'adressent à un public conservateur moyennement cultivé, lui offrant des clés de lecture de l'actualité internationale, et parce qu'il a ainsi contribué à 
faire accepter au grand public la modernisation ou l'« occidentalisation » de la République fédérale, que Mehnert, quoique conservateur, a acquis le statut d'intellectuel ouestallemand. Michael Kohlstruck souligne la nécessité d'élargir la définition d'intellectuel dans un sens sociologique, définition tournée vers les acteurs et la diffusion d'idées et de valeurs, qu'elles soient de gauche ou plus conservatrices.

Le volume se clôt sur une contribution du politologue Hans-Manfred Bock qui rend compte des tentatives menées ces trois dernières décennies par des universitaires pour dépasser le système auto-référentiel, dans lequel les intellectuels définissaient eux-mêmes ce qui les caractérisait, et proposer une définition historico-sociologique du rôle des intellectuels. Révélant la fragilité de valeurs supposées universelles, le post-fondamentalisme a sérieusement entamé le socle sur lequel reposaient l'autorité et la légitimité, partant les formes de recrutement et d'intervention des intellectuels correspondant au modèle qui a perduré de Zola à Sartre. À l'intellectuel "universel » succède ainsi, d'après Foucault, l'intellectuel "spécifique » qui, dans des situations précises, aurait pour tâche de constituer des réseaux de résistance au pouvoir, au capital, etc. La sociologie des intellectuels de Bourdieu, qui présente la position d'autorité de l'intellectuel comme le produit d'interactions conflictuelles dans un champ social et non comme le résultat d'une auto-proclamation ou de la reconnaissance sociale de son autorité, a fortement contribué à démythologiser cet acteur social et culturel. Si Jean-François Lyotard remet en question l'existence des intellectuels en leur attribuant tout au plus un rôle d'intervenants ponctuels auprès des citoyens sur des questions précises, Pierre Nora et Marcel Gauchet, dans le périodique Le Débat, nourrissent la discussion internationale sur la transformation ou le déclin des intellectuels dans le présent et à l'avenir.

Cet ouvrage offre une galerie de portraits d'intellectuels d'une grande richesse et convainc aisément de la fluidité des frontières entre intellectuels et anti-intellectuels. Dans ce sens, on s'étonne même de voir figurer le portrait de Klaus Mehnert dans la partie dédiée aux anti-intellectuels, car Mehnert apparaît comme un intellectuel, du moins par la profession, qui a fait le choix de s'adresser à un public moyennement cultivé mais intéressé en recourant à des formes de vulgarisation. On ne voit pas en quoi Mehnert était anti-intellectuel et sans doute la contribution à son sujet figure-t-elle dans cette partie pour équilibrer les deux parties du volume. La symétrie dans l'organisation du volume semble alors un peu artificielle. On peut également regretter que certaines contributions, si intéressants que soient les portraits qu'elles proposent, soient bien peu aux prises avec la définition de l'intellectuel.

La contribution d'Anne Kwaschik sur la naissance du modèle de l'intellectuel apporte des éclairages d'une prime importance sur le modèle auquel se réfèrent toutes les réflexions sur la définition de l'intellectuel, éclairages d'autant plus appréciables que l'introduction de l'ouvrage ne propose pas de cadre théorique situant son objet dans la recherche existante. Pour cette même raison, on apprécie particulièrement les réflexions de Hans-Manfred Bock qui dessine les lignes de force des discussions des trois dernières décennies sur la définition et le rôle des intellectuels. Incontestablement, cet ouvrage apporte sa pierre à l'édifice de ces discussions et il trouvera sa place dans l'ensemble de travaux sur les intellectuels en Allemagne, un champ d'étude qui est encore loin d'être épuisé. 